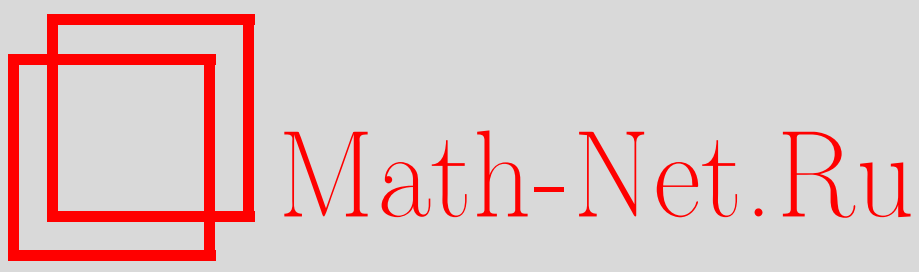

V. V. Aseev, Adherence of the images of points under multivalued quasimöbius mappings, Sibirsk. Mat. Zh., 2020, Volume 61, Number 3, 499-512

DOI: https://doi.org/10.33048/smzh.2020.61.302

Use of the all-Russian mathematical portal Math-Net.Ru implies that you have read and agreed to these terms of use http://www . mathnet.ru/eng/agreement

Download details:

IP: 52.87 .193 .239

April 26, 2023, 10:03:29

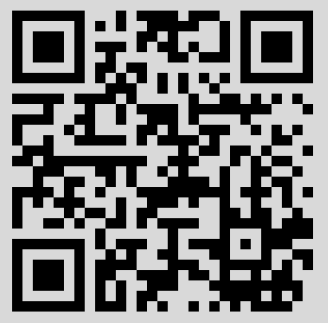


Сибирский математический журнал Май-июнь, 2020. Том 61, № 3

УДК 517.54

\title{
О ПРИМЫКАНИИ ОБРАЗОВ ТОЧЕК У МНОГОЗНАЧНЫХ КВАЗИМЁБИУСОВЫХ ОТОБРАЖЕНИЙ
}

\author{
B. В. Асеев
}

\begin{abstract}
Аннотация. Продолжено изучение многозначных отображений со свойством BAD (bounded angular distortion), начатое в работах автора в 2018 г. С использованием техники ультрафильтров получено полное описание областей взаимного примыкания образов точек при отображениях класса BAD. Построен пример, иллюстрирующий все возможные случаи в общей формуле для области примыкания.
\end{abstract}

DOI 10.33048/smzh.2020.61.302

Ключевые слова: многозначное отображение, мёбиусова структура, мёбиусово пространство, птолемеева полуметрика, квазиконформное отображение, квазимёбиусово отображение, фильтр, ультрафильтр.

Статья завершает исследование взаимного расположения образов точек при многозначных отображениях с обобщенным свойством квазимёбиусовости (свойство BAD), начатое автором в статьях 2018 г., и является непосредственным продолжением работы [1]. Учитывая, что история вопроса и его проблематика, мотивация к введению и изучению многозначных отображений со свойством BAD [1-5], а также их связь с квазимёбиусовыми отображениями [6] и отображениями с ограниченным искажением в смысле Ю. Г. Решетняка [7] достаточно освещены в [1], ограничимся здесь лишь списком основных определений и теорем, необходимых для понимания излагаемых результатов.

\section{Введение}

Согласно С. В. Буяло [8] мёбиусовой структурой на множестве $X$, содержащем не менее четырех точек, называется семейство $\mathscr{M}$ всех полуметрик на $X$, связанных отношением мёбиусовой эквивалентности $\rho \sim \sigma$, означающим, что

$$
\frac{\rho\left(x_{1}, x_{2}\right) \rho\left(x_{3}, x_{4}\right)}{\rho\left(x_{1}, x_{3}\right) \rho\left(x_{2}, x_{4}\right)}=\frac{\sigma\left(x_{1}, x_{2}\right) \sigma\left(x_{3}, x_{4}\right)}{\sigma\left(x_{1}, x_{3}\right) \sigma\left(x_{2}, x_{4}\right)}
$$

для любой тетрады (т. е. четверки попарно различных точек) в $X$. Пару $(X, \mathscr{M})$ называем мёбиусовым пространством. Если хотя бы одна (а значит, любая) полуметрика $\rho \in \mathscr{M}$ удовлетворяет неравенству Птолемея

$$
\rho\left(x_{1}, x_{2}\right) \rho\left(x_{3}, x_{4}\right)+\rho\left(x_{1}, x_{4}\right) \rho\left(x_{2}, x_{3}\right) \geq \rho\left(x_{1}, x_{3}\right) \rho\left(x_{2}, x_{4}\right),
$$

Работа выполнена при поддержке бюджетной темы НИР (номер проекта 0314-20190007).

(c) 2020 Асеев B. B. 
то мёбиусово пространство $(X, \mathscr{M})$ называем птолемеевым. Все рассматриваемые в этой статье метрики и полуметрики считаются по умолчанию птолемеевыми. Как показано в [3, теорема 1.4], в любой птолемеевой структуре $\mathscr{M}$ имеется ограниченная метрика $\rho \in \mathscr{M}, \rho(.,) \leq$.1 . При этом все метрики из $\mathscr{M}$ порождают одну и ту же топологию в $X$ (см. [4, утверждение 1.2$])$, которую называем метрической топологией в птолемеевом мёбиусовом пространстве $(X, \mathscr{M})$ и по умолчанию считаем, что $X$ наделено именно этой топологией. В птолемеевом мёбиусовом пространстве $(X, \mathscr{M})$ птолемеева характеристика тетрадъ

$$
\alpha\left(a_{1}, a_{2} ; b_{1}, b_{2}\right):=\frac{\rho\left(a_{1}, a_{2}\right) \rho\left(b_{1}, b_{2}\right)}{\rho\left(a_{1}, b_{1}\right) \rho\left(a_{2}, b_{2}\right)+\rho\left(a_{1}, b_{2}\right) \rho\left(a_{2}, b_{1}\right)} \leq 1
$$

не зависит от выбора полуметрики $\rho \in \mathscr{M}$, т. е. является мёбиусово инвариантной величиной. Обобщенным углом называем любую четверку множеств $\Psi=\left(A_{1}, A_{2} ; B_{1}, B_{2}\right)$, у которой $B_{1} \neq \varnothing \neq B_{2}$ и $B_{1} \cup B_{2}$ содержит две различные точки. Величиной обобщенного угла $\Psi=\left(A_{1}, A_{2} ; B_{1}, B_{2}\right)$ в птолемеевом мёбиусовом пространстве $(X, \mathscr{M})$ является число

$$
\alpha(\Psi)=\alpha\left(A_{1}, A_{2} ; B_{1}, B_{2}\right):=\inf _{a_{1} \in A_{1} ; a_{2} \in A_{2}}\left(\sup _{b_{1} \in B_{1} ; b_{2} \in B_{2}} \alpha\left(a_{1}, a_{2} ; b_{1}, b_{2}\right)\right) \leq 1,
$$

не зависящее от выбора полуметрики $\rho \in \mathscr{M}$. Тетрада $\left(a_{1}, a_{2} ; b_{1}, b_{2}\right)$ рассматривается как частный случай обобщенного угла.

Многозначное отображение $F: X \rightarrow 2^{Y}$ называем гиперингективным, если $\left(x_{1} \neq x_{2}\right) \Rightarrow\left(F\left(x_{1}\right) \cap F\left(x_{2}\right)=\varnothing\right)$. Гиперинъективное отображение переводит обобщенные углы в обобщенные углы.

В птолемеевых мёбиусовых пространствах $(X, \mathscr{M}),\left(Y, \mathscr{M}^{\prime}\right)$ гиперинъективное отображение $F: X \rightarrow 2^{Y}$ обладает свойством BAD (bounded angular distortion), если существует положительная неубывающая функция $\eta:(0,1] \rightarrow$ $R_{+}$(контрольная функция), при которой

$$
\alpha\left(F\left(a_{1}\right), F\left(a_{2}\right) ; F\left(b_{1}\right), F\left(b_{2}\right)\right) \geq \eta\left(\alpha\left(a_{1}, a_{2} ; b_{1}, b_{2}\right)\right)
$$

для любой тетрады $\left(a_{1}, a_{2} ; b_{1}, b_{2}\right)$ в $X$. В этом случае также используем запись $F \in \eta$-BAD. Заметим, что если две контрольные функции $\eta_{1}:(0,1] \rightarrow R_{+}$и $\eta_{2}:(0,1] \rightarrow R_{+}$связаны соотношением $\eta_{2}(t) \leq \eta_{1}(t)$ при всех $t \in(0,1]$, то из $F \in \eta_{1}$-BAD следует $F \in \eta_{2}$-BAD. Поэтому, заменив $\eta(t)$ функцией $\min \{\eta(t), 1\}$, можно без нарушения общности считать, что контрольная функция $\eta$ в формуле $F \in \eta$-BAD удовлетворяет неравенству $\eta(t) \leq 1$ при всех $t \in(0,1]$.

\section{$\S 1$. Некоторые свойства отображений класса BAD}

Непустые непересекающиеся множества $A, B$ в метрическом пространстве $(X, \rho)$ примыкают друг к другу, если inf $\{\rho(a, b): a \in A, b \in B\}=0$.

1.1. Утверждение [1, утверждение 2.4]. Если непустые непересекающиеся множества $A, B \subset X$ в птолемеевом мёбиусовом пространстве $(X, \mathscr{M})$ примыкают друг к другу в некоторой метрике из $\mathscr{M}$, то они примыкают друг к другу в любой ограниченной метрике из $\mathscr{M}$.

Образ точки при многозначном отображении будем называть толстой точкой. 
1.2. Теорема [3, теорема 6.1]. Пусть $F: X \rightarrow 2^{Y}-$ многозначное отображение класса BAD в птолемеевых мёбиусовых пространствах $(X, \mathscr{M})$ и $\left(Y, \mathscr{M}^{\prime}\right)$. Тогда для любой метрики $\sigma \in \mathscr{M}^{\prime}$ реализуется одна из следующих ситуаций:

(I) в $Y$ нет толстых точек, примыкающих друг к другу;

(II) существует пара $F(p), F(q) \subset Y$ толстых точек, не примыкающих друг к другу и таких, что любая толстая точка, отличная от $F(q)$ и $F(p)$, примыкает к $F(p)$;

(III) все толстые точки в $Y$ примыкают друг к другу.

Свойство примыкания толстых точек не является мёбиусово инвариантным, но оно не зависит от выбора ограниченной метрики в мёбиусовой структуpe $\left(Y, \mathscr{M}^{\prime}\right)$. При этом альтернатива (I)-(III) остается справедливой при любой метрике (даже неограниченной) из $\mathscr{M}^{\prime}$.

1.3. Теорема [3, теорема 6.2]. Пусть $F: X \rightarrow 2^{Y}$ - многозначное отображение класса $B A D$ в птолемеевых мёбиусовых пространствах $(X, \mathscr{M})$ и $\left(Y, \mathscr{M}^{\prime}\right)$. Тогда для левого обратного отображения $f: F(X) \rightarrow X$ в любой точке $y_{0} \in$ $F(X)$ реализуется одна из ситуаций:

(I) отображение $f$ непрерывно в точке $y_{0}$;

(II) в любой сколь угодно малой окрестности точки $y_{0}$ отображение $f$ принимает все значения из $X$, кроме, может быть, одного.

Стоит заметить, что поведение функции $f$ в этой теореме аналогично поведению аналитической функции в окрестности изолированной особой точки.

Ситуация (I) в теореме 1.2 подробно изучена в [1]. В частности, получена

1.4. Теорема $[1$, теоремы $3.2,3.5]$. Пусть птолемеево мёбиусово пространство $\left(Y, \mathscr{M}^{\prime}\right)$ компактно в своей метрической топологии, многозначное отображение $F: X \rightarrow 2^{Y}$ птолемеева мёбиусова пространства $(X, \mathscr{M})$ обладает свойством $\omega$-BAD и все толстые точки замкнуты в $Y$. Тогда

(i) для любой точки $x_{0} \in X$ и любой открытой окрестности $V$ толстой точки $F\left(x_{0}\right)$ существует окрестность $U$ точки $x_{0}$ такая, что $F(U) \subset V$;

(ii) если $x_{0}$ - предельная точка в $X$, то существует топологический предел $L\left(x_{0}\right)=\operatorname{Lim}_{x \rightarrow x_{0} ; x \neq x_{0}} F(x) \subset F\left(x_{0}\right) ;$ кроме того, для любой метрики $\sigma \in \mathscr{M}^{\prime}$ и инверсной метрики $h(z, w):=\sigma(z, w) /(\sigma(z, q) \sigma(w, q))$ с полюсом $q \in F(X) \backslash F\left(x_{0}\right)$ в любой точке $p \in F\left(x_{0}\right)$ справедлива оценка

$$
\operatorname{dist}_{h}\left(p, L\left(x_{0}\right)\right) \leq \operatorname{diam}_{h}\left(L\left(x_{0}\right)\right) /(2 \omega(1)) ;
$$

отображение $F^{0}(x)=\{F(x)$ в изолированной точке; $L(x)$ в предельной точке $\}$ имеет свойство $\omega$-BAD c той же контрольной функцией $\omega$.

В данной статье исследованы ситуации (II) и (III) в теореме 1.2 и дано полное описание характера примыкания толстых точек в общем случае.

\section{$\S 2$. Области примыкания}

Изучение примыкания толстых точек к фиксированной толстой точке $A=$ $F(x)$ будет проведено в терминах ультрафильтров на множестве $A$.

2.1. ОПредеЛЕниЕ. Пусть на множестве $X$, содержащем непустые подмножества $A$ и $B$, задана метрика $\rho$. Для произвольного $\varepsilon>0$ положим $U_{\rho}^{\varepsilon}(A ; B):=\{x \in A: \rho(x, B)<\varepsilon\}$. Если $B$ примыкает к $A$ в метрике $\rho$, то семейство подмножеств $\left(U_{\rho}^{\varepsilon}(A ; B)\right)_{\varepsilon>0}$ образует базис фильтра $R_{\rho}(A ; B)$ в $A$. Областъю примыкания множества $B$ к множеству $A$ назовем семейство $\mathscr{N}_{\rho}(A ; B)$ 
всех ультрафильтров в $A$, мажорирующих фильтр $R_{\rho}(A ; B)$. При этом полагаем $\mathscr{N}_{\rho}(A ; B)=\varnothing$ в том и только в том случае, когда $B$ не примыкает к $A$.

2.2. Лемма. Пусть $(X, \mathscr{M})$ - птолемеева мёбиусова структура и непустые подмножества $A, B \subset X$ примыкают друг к другу в некоторой метрике $\rho \in \mathscr{M}$. Тогда (см. [1, утверждение 2.4]) они примыкают друг к другу в любой ограниченной метрике из $\mathscr{M}$. При этом для любых ограниченных метрик $\rho_{1}, \rho_{2} \in \mathscr{M}$

$$
\mathscr{N}_{\rho_{1}}(A ; B)=\mathscr{N}_{\rho_{2}}(A ; B),
$$

т. е. область примыкания $\mathscr{N}(A ; B):=\mathscr{N}_{\rho}(A ; B)$ не зависит от выбора ограниченной метрики $\rho \in \mathscr{M}$.

ДокАзАтельство. Допустим, нашлось такое $\delta>0$, что для любого натурального $n \in \mathbf{N}$ имеется точка $x_{n} \in U_{\rho_{1}}^{1 / n}(A ; B) \backslash U_{\rho_{2}}^{\delta}(A ; B)$. Последовательность $\left\{x_{n}\right\}$ образует множество $A_{0} \subset A$, не примыкающее к $B$ в метрике $\rho_{2}$. Тогда в силу [1, утверждение 2.4] $A_{0}$ не примыкает к $B$ и в метрике $\rho_{1}$. Это противоречит тому, что $\rho_{1}\left(x_{n}, B\right)<1 / n \rightarrow 0$ при $n \rightarrow \infty$.

Следовательно, найдется такой номер $n(\delta) \in \mathbf{N}$, что при всех $n>n(\delta)$ справедливо включение $U_{\rho_{1}}^{1 / n}(A ; B) \subset U_{\rho_{2}}^{\delta}$, и это означает, что всякое множество из базиса фильтра $R_{\rho_{2}}(A ; B)$ содержит множество из базиса фильтра $R_{\rho_{1}}(A ; B)$, т. е. (см. $\left[9\right.$, гл. $1, \S 6.3$, предложение 4]) фильтр $R_{\rho_{1}}(A ; B)$ мажорирует фильтр $R_{\rho_{2}}(A ; B)$. Так как ограниченные метрики $\rho_{1}$ и $\rho_{2}$ в этом рассуждении равноправны, фильтр $R_{\rho_{2}}(A ; B)$ мажорирует фильтр $R_{\rho_{1}}(A ; B)$. Значит, эти фильтры совпадают, поэтому $\mathscr{N}_{\rho_{1}}(A ; B)=\mathscr{N}_{\rho_{2}}(A ; B)$. Лемма доказана.

Наиболее простую ситуацию демонстрирует

2.3. Утверждение. Пусть $X$ компактно в метрической топологии, порожденной птолемеевой мёбиусовой структурой на $X$, и непустые множества $A, B \subset X$ примыкают друг к другу. Тогда область примыкания $\mathscr{N}(A ; B)$ состоит из всех ультрафильтров в $A$, имеющих предел в $\bar{A} \cap \bar{B}$.

ДокаЗАтельство. В силу компактности $X$ множество $\bar{A} \cap \bar{B}$ непустое. Покажем, что любой фильтр $\mathbf{F}$ в $A$, мажорирующий фильтр $R(A ; B)$, имеет точку прикосновения в $\bar{A} \cap \bar{B}$. Действительно, в противном случае каждая точка $x \in \bar{A} \cap \bar{B}$, не будучи точкой прикосновения для $\mathbf{F}$, имела бы окрестность $U(x)$, не пересекающуюся с некоторым множеством $F(x) \in \mathbf{F}$. Взяв конечное покрытие $\left\{U\left(x_{j}\right): j=1, \ldots, m\right\}$ компактного множества $\bar{A} \cap \bar{B}$, получим элемент $F=\bigcap_{j=1}^{m} F\left(x_{j}\right) \in \mathbf{F}$, который не пересекается с $U=\bigcup_{j=1}^{m} U\left(x_{j}\right)$ - окрестностью множества $\bar{A} \cap \bar{B}$. Но $\mathbf{F}$ мажорирует $R(A, B)$ и должен содержать множество $U \cap A$, в частности $(U \cap A) \subset F$, что противоречит $F \cap U=\varnothing$.

В частности, любой ультрафильтр $\mathbf{F} \in \mathscr{N}(A ; B)$ имеет точку прикосновения (а значит, и предел, см. [9, гл. $1, \S 7$, следствие]) в $\bar{A} \cap \bar{B}$.

Обратно, если $x \in \bar{A} \cap \bar{B}$, то любой ультрафильтр $\mathbf{F}$ в $A$, имеющий предел $x$, мажорирует фильтр, порожденный системой окрестностей точки $x$ в $A$. Значит, он мажорирует фильтр $R(A ; B)$, т. е. $\mathbf{F} \in \mathscr{N}(A ; B)$.

Утверждение доказано.

2.4. Утверждение. Пусть непустые множества $A, B, C$ в птолемеевом мёбиусовом пространстве $(X, \mathscr{M})$ таковы, что $\mathscr{N}(A ; B) \neq \varnothing \neq \mathscr{N}(A ; C)$. Тогда

$$
(\mathscr{N}(B ; C)=\varnothing) \Rightarrow(\mathscr{N}(A ; B) \cap \mathscr{N}(A ; C)=\varnothing) .
$$


ДокАЗАТЕЛЬСтво. Фиксируем ограниченную метрику $\rho \in \mathscr{M}$. Пусть $\mathscr{N}(B ; C)=\varnothing$. Тогда $\delta=\inf \{\rho(u, v): u \in B, v \in C\}>0$. Множества $U_{\rho}^{\delta / 3}(A ; B)$ и $U_{\rho}^{\delta / 3}(A ; C)$ не пересекаются, ибо в противном случае для их общей точки $x$ нашлись бы такие $u \in B$ и $v \in C$, что $\rho(x, u)<\delta / 3$ и $\rho(x, v)<\delta / 3$, и это привело бы к противоречию: $\rho(u, v) \leq \rho(u, x)+\rho(x, v)<2 \delta / 3<\delta$. Так как $U_{\rho}^{\delta / 3}(A ; B) \in R(A ; B)$ и $U_{\rho}^{\delta / 3}(A ; C) \in R(A ; C)$, не существует фильтра (и ультрафильтра), мажорирующего оба фильтра $R(A ; B)$ и $R(A ; C)$. Это означает, что области примыкания $\mathscr{N}(A ; B)$ и $\mathscr{N}(A ; C)$ не могут иметь общего элемента. Утверждение доказано.

\section{§ 3. Структура области примыкания толстых точек}

В этом параграфе $\left(X, \mathscr{M}^{\prime}\right)$ и $(Y, \mathscr{M})$ - птолемеевы мёбиусовы пространства и отображение $F: X \rightarrow 2^{Y}$ обладает свойством $\eta$-BAD.

3.1. Лемма. Если для попарно различных точек $a, b, c, d \in X$ и их образов $A=F(a), B=F(b), C=F(c), D=F(d)$ области примыкания $\mathscr{N}(A ; B)$ и $\mathscr{N}(A ; D)$ непусты, то

$$
\mathscr{N}(A ; B) \backslash \mathscr{N}(A ; C)=\mathscr{N}(A ; D) \backslash \mathscr{N}(A ; C) .
$$

ДокАЗАТЕЛЬСтво проведем для случая $(1 \mathrm{a}) \mathscr{N}(A ; C) \neq \varnothing$ и случая (2а) $\mathscr{N}(A ; C)=\varnothing$, отмечая те пункты, где требуется различать эти случаи.

Фиксируем ограниченную метрику $\sigma \in \mathscr{M}$. Толстые точки $B, D$ в (3.1.1) равноправны, поэтому достаточно установить включение

$$
\mathscr{N}(A ; B) \backslash \mathscr{N}(A ; C) \subset \mathscr{N}(A ; D) \backslash \mathscr{N}(A ; C)
$$

в случае $(1 \mathrm{a})$ и включение

$$
\mathscr{N}(A ; B) \subset \mathscr{N}(A ; D)
$$

в случае $(2 \mathrm{a})$.

Допустим от противного, что на множестве $A$ имеется ультрафильтр $\mathbf{Z}$, мажорирующий $R_{\sigma}(A ; B)$, но не мажорирующий фильтр $R_{\sigma}(A ; D)$ (а также не мажорирующий фильтр $R_{\sigma}(A ; C)$ в случае $\left.(1 \mathrm{a})\right)$.

Фраза «фильтр $\mathbf{Z}$ не мажорирует фильтр $R_{\sigma}(A ; D) »$ означает, что существует элемент $U_{\sigma}^{\delta^{\prime}}(A ; D)=\left\{u \in A: \sigma(u, D)<\delta^{\prime}\right\}$ базиса фильтра $R_{\sigma}(A ; D)$, не входящий в $\mathbf{Z}$. Так как $\mathbf{Z}$ - ультрафильтр, дополнение в $A$ к этому элементу (т. е. множество $\left\{u \in A: \sigma(u, D) \geq \delta^{\prime}\right\}$ ) входит в $\mathbf{Z}$ (см. [9, гл. $1, \S 6.4$, предложение 5]). Аналогично фраза «ультрафильтр $\mathbf{Z}$ не мажорирует фильтр $R_{\sigma}(A ; C)$ » (в случае (1a)) означает, что для некоторого $\delta^{\prime \prime}>0$ множество $\{u \in A: \sigma(u, C) \geq$ $\left.\delta^{\prime \prime}\right\}$ принадлежит $\mathbf{Z}$. В случае (2а) толстые точки $A$ и $C$ не примыкают друг к другу и в качестве $\delta^{\prime \prime}$ можно взять $\delta^{\prime \prime}=\inf \{\sigma(u, v): u \in A, v \in C\}>0$. Положим $\delta_{0}=\min \left\{\delta^{\prime}, \delta^{\prime \prime}\right\}>0$. Тогда из соотношений

$$
\begin{gathered}
\left\{u \in A: \sigma(u, C) \geq \delta^{\prime \prime}\right\} \subset\left\{u \in A: \sigma(u, C) \geq \delta_{0}\right\} \in \mathbf{Z} \quad \text { в случае (1a), } \\
\left\{u \in A: \sigma(u, D) \geq \delta^{\prime}\right\} \subset A=\left\{u \in A: \sigma(u, C) \geq \delta_{0}\right\} \in \mathbf{Z} \quad \text { в случае (2a) }
\end{gathered}
$$

и

$$
\left\{u \in A: \sigma(u, D) \geq \delta^{\prime}\right\} \subset\left\{u \in A: \sigma(u, D) \geq \delta_{0}\right\} \in \mathbf{Z}
$$

следует, что в обоих случаях $(1 \mathrm{a})$ и $(2 \mathrm{a})$

$$
\left\{u \in A: \sigma(u, C \cup D) \geq \delta_{0}\right\} \in \mathbf{Z} .
$$


Фраза «ультрафильтр $\mathbf{Z}$ мажорирует фильтр $R_{\sigma}(A ; B) »$ означает, что при любом $\varepsilon>0$ элемент $\{u \in A: \sigma(u, B)<\varepsilon\}$ базиса фильтра $R_{\sigma}(A ; B)$ принадлежит Z. С учетом (3.1.3) при любом $\varepsilon>0$ множество

$$
\begin{aligned}
S(\varepsilon):=\{u \in A: \sigma(u, B)<\varepsilon\} \cap & \left\{u \in A: \sigma(u, D \cup C) \geq \delta_{0}\right\} \\
& =\left\{u \in A: \sigma(u, B)<\varepsilon, \sigma(u, D \cup C) \geq \delta_{0}\right\} \in \mathbf{Z}
\end{aligned}
$$

непустое. Значит, при любом $\varepsilon>0$ имеются точки $u_{1}^{(\varepsilon)} \in A$ и $u_{2}^{(\varepsilon)} \in B$, у которых $\sigma\left(u_{1}^{(\varepsilon)}, u_{2}^{(\varepsilon)}\right)<\varepsilon$, но $\sigma\left(u_{1}^{(\varepsilon)}, C \cup D\right) \geq \delta_{0}$. В частности, для любых $v_{1} \in C, v_{2} \in D$ выполняются оценки $\sigma\left(u_{1}^{(\varepsilon)}, v_{1}\right) \geq \delta_{0}, \sigma\left(u_{1}^{(\varepsilon)}, v_{2}\right) \geq \delta_{0}$ и неравенство

$$
\begin{aligned}
\alpha\left(u_{1}^{(\varepsilon)}, u_{2}^{(\varepsilon)} ; v_{1}, v_{2}\right)=\frac{\sigma\left(u_{1}^{(\varepsilon)}, u_{2}^{(\varepsilon)}\right) \sigma\left(v_{1}, v_{2}\right)}{\sigma\left(u_{1}^{(\varepsilon)}, v_{1}\right) \sigma\left(u_{2}^{(\varepsilon)}, v_{2}\right)+\sigma\left(u_{1}^{(\varepsilon)}, v_{2}\right) \sigma\left(u_{2}^{(\varepsilon)}, v_{1}\right)} \\
\leq \frac{\varepsilon}{\delta_{0}} \cdot \frac{\sigma\left(v_{1}, v_{2}\right)}{\sigma\left(u_{2}^{(\varepsilon)}, v_{1}\right)+\sigma\left(u_{2}^{(\varepsilon)}, v_{2}\right)} \leq \frac{\varepsilon}{\delta_{0}} .
\end{aligned}
$$

Следовательно,

$$
\sup _{v_{1} \in C ; v_{2} \in D} \alpha\left(u_{1}^{(\varepsilon)}, u_{2}^{(\varepsilon)} ; v_{1}, v_{2}\right) \leq \varepsilon / \delta_{0}
$$

и для образа $F(\Psi)=(A, B ; C, D)$ тетрады $\Psi=(a, b ; c, d)$ получаем оценку

$$
\begin{aligned}
\alpha(F(\Psi))= & \inf _{u_{1} \in A ; u_{2} \in B}\left(\sup _{v_{1} \in C ; v_{2} \in D} \alpha\left(u_{1}, u_{2} ; v_{1}, v_{2}\right)\right) \\
& \leq \sup _{v_{1} \in C ; v_{2} \in D} \alpha\left(u_{1}^{(\varepsilon)}, u_{2}^{(\varepsilon)} ; v_{1}, v_{2}\right) \leq \varepsilon / \delta_{0},
\end{aligned}
$$

выполняющуюся при любом $\varepsilon>0$. Но это противоречит свойству $\eta$-BAD отображения $F$, согласно которому $\alpha(F(\Psi)) \geq \eta(\alpha(\Psi))>0$. Полученное противоречие завершает доказательство леммы.

Теперь можно существенно уточнить ситуацию (II) в теореме 1.2.

3.2. Теорема. Пусть в птолемеевых мёбиусовых пространствах $\left(X, \mathscr{M}^{\prime}\right)$ и $(Y, \mathscr{M})$ задано отображение $F: X \rightarrow 2^{Y}$ со свойством BAD. Пусть в некоторой ограниченной метрике $\sigma \in \mathscr{M}^{\prime}$ имеются толстые точки, примыкающие друг к другу, и толстые точки, не примыкающие друг к другу. Тогда возможны только две ситуации:

(II.a) имеется единственная толстая точка $Q$, не примыкающая ни к одной из остальных толстых точек, при этом для любых попарно различных толстых точек $P, A, B$, отличных от $Q, \mathscr{N}(A ; B) \neq \varnothing$ и

$$
\mathscr{N}(P ; A)=\mathscr{N}(P ; B) \neq \varnothing ;
$$

(II.b) имеется единственная пара не примыкающих друг к другу толстых точек $P$ и $Q$, при этом для любой пары различных толстых точек $A$ и $B$, отличных от $P$ и $Q, \mathscr{N}(A ; B) \neq \varnothing$ и выполняются равенства (3.2.1) и

$$
\mathscr{N}(Q ; A)=\mathscr{N}(Q ; B) \neq \varnothing .
$$

ДокАзАтЕльство. Так как реализуется ситуация (II) в теореме 1.2, существуют толстые точки $P$ и $Q$, не примыкающие друг к другу и такие, что любая другая толстая точка примыкает к $P$. Тогда для любых толстых точек $A$ и $B$, 
отличных от $Q, \mathscr{N}(P ; A) \neq \varnothing \neq \mathscr{N}(P ; B)$ и $\mathscr{N}(P ; Q)=\varnothing$. Лемма 3.1 дает равенство (3.2.1)

$$
\mathscr{N}(P ; A)=\mathscr{N}(P ; A) \backslash \mathscr{N}(P ; Q)=\mathscr{N}(P ; B) \backslash \mathscr{N}(P ; Q)=\mathscr{N}(P ; B),
$$

а утверждение $2.4-$ соотношение $\mathscr{N}(A ; B) \neq \varnothing$.

Итак, все толстые точки, отличные от $Q$, примыкают друг к другу. Если ни одна из них не примыкает к $Q$, то реализуется ситуация (II.a).

Рассмотрим случай, когда имеется толстая точка $A$, отличная от $P, Q$ и примыкающая к $Q$. Тогда $\mathscr{N}(A ; Q) \neq \varnothing \neq \mathscr{N}(A ; P)$, и лемма 3.1 дает равенство

$$
\mathscr{N}(A ; P) \backslash \mathscr{N}(A ; B)=\mathscr{N}(A ; Q) \backslash \mathscr{N}(A ; B) .
$$

Так как в силу утверждения $2.4 \mathscr{N}(A ; P) \cap \mathscr{N}(A ; Q)=\varnothing$, это равенство возможно лишь в том случае, когда $\mathscr{N}(A ; P) \subset \mathscr{N}(A ; B)$ и

$$
\varnothing \neq \mathscr{N}(A ; Q) \subset \mathscr{N}(A ; B) .
$$

Предположение $\mathscr{N}(B ; Q)=\varnothing$ приводит в силу утверждения 2.4 к равенству $\mathscr{N}(A ; B) \cap \mathscr{N}(A ; Q)=\varnothing$, противоречащему (3.2.3). Значит, любая толстая точка $B$, отличная от $P$ и $Q$, примыкает как к $P$, так и к $Q$. При этом по лемме 3.1

$$
\mathscr{N}(Q ; A) \backslash \mathscr{N}(Q ; P)=\mathscr{N}(Q ; B) \backslash \mathscr{N}(Q ; P)
$$

и в силу равенства $\mathscr{N}(Q ; P)=\varnothing$ получаем (3.2.2). Заметим, что в этом случае $P$ и $Q-$ единственная пара толстых точек, не примыкающих друг к другу, и точки $P, Q$ в этой паре равноправны. Таким образом, реализуется ситуация (II.b).

Теорема доказана.

Теперь можно снять в лемме 3.1 ограничение $\mathscr{N}(A ; B) \neq \varnothing \neq \mathscr{N}(A ; D)$.

\section{3. Лемма. Равенство}

$$
\mathscr{N}(A ; B) \backslash \mathscr{N}(A ; C)=\mathscr{N}(A ; D) \backslash \mathscr{N}(A ; C)
$$

выполняется для любых попарно различных толстых точек $A, B, C, D$.

ДокАЗАТЕЛЬСтво. С учетом леммы 3.1 осталось рассмотреть случай, когда $\mathscr{N}(A ; B)=\varnothing$ или $\mathscr{N}(A ; D)=\varnothing$. Так как $B$ и $D$ в (3.3.1) равноправны, достаточно ограничиться случаем $\mathscr{N}(A ; D)=\varnothing$. Тогда требуемое равенство (3.3.1) имеет вид

$$
\mathscr{N}(A ; B) \backslash \mathscr{N}(A ; C)=\varnothing
$$

и тривиально верно при $\mathscr{N}(A ; B)=\varnothing$.

Пусть теперь $\mathscr{N}(A ; B) \neq \varnothing$. Среди толстых точек $A, B, C, D$ есть пара $A, B$ примыкающих друг к другу и пара $A, D$ не примыкающих друг к другу толстых точек, т. е. выполнены условия теоремы 3.2 с $P=A, Q=D$. По этой теореме все толстые точки, отличные от $D$, примыкают к $A$ и имеют в $A$ совпадающие области примыкания, т. е. $\mathscr{N}(A ; B)=\mathscr{N}(A ; C)$ - требуемое равенство (3.3.2).

Лемма доказана.

Основным результатом данной статьи является 
3.4. Теорема. Пусть в птолемеевых мёбиусовых пространствах $\left(X, \mathscr{M}^{\prime}\right)$ и $(Y, \mathscr{M})$ задано отображение $F: X \rightarrow 2^{Y}$ со свойством BAD. Тогда для любой точки $a \in X$ справедлива формула

$$
\bigcup_{x \in X \backslash\{a\}} \mathscr{N}(F(a) ; F(x))=\bigcup_{b \in X \backslash\{a\}}\left(\bigcap_{x \in X \backslash\{a, b\}} \mathscr{N}(F(a) ; F(x))\right) .
$$

ДоказАтЕльство. Достаточно установить включение

$$
\bigcup_{x \in X \backslash\{a\}} \mathscr{N}(F(a) ; F(x)) \subset \bigcup_{b \in X \backslash\{a\}}\left(\bigcap_{x \in X \backslash\{a, b\}} \mathscr{N}(F(a) ; F(x))\right),
$$

так как обратное включение очевидно.

Для толстой точки $C$, отличной от $A=F(a)$, и ультрафильтра $\mathbf{Z} \in \mathscr{N}(A ; C)$ возможны две ситуации.

(b1) $\mathbf{Z} \in \mathscr{N}(A ; F(x))$ для любой толстой точки $F(x)$, отличной от $A$. Тогда (см. [10, гл. II, $\S 4.3$, предложение 2])

$$
\begin{array}{r}
\mathbf{Z} \in \bigcap_{x \in X \backslash\{a\}} \mathscr{N}(A ; F(x))=\bigcap_{b \in X \backslash\{a\}}\left(\bigcap_{x \in X \backslash\{a, b\}} \mathscr{N}(A ; F(x))\right) \\
\subset \bigcup_{b \in X \backslash\{a\}}\left(\bigcap_{x \in X \backslash\{a, b\}} \mathscr{N}(A ; F(x))\right) .
\end{array}
$$

(b2) Имеется толстая точка $B=F(b) \neq A$, для которой $\mathbf{Z} \notin \mathscr{N}(A ; B)$. Так как $\mathbf{Z} \in \mathscr{N}(A ; C) \backslash \mathscr{N}(A ; B)$, (по лемме 3.3$) \mathbf{Z} \in \mathscr{N}(A ; F(x)) \backslash \mathscr{N}(A ; B)$ для любой толстой точки $F(x)$, отличной от $A$ и $B$, т. е.

$$
\mathbf{Z} \in \bigcap_{x \in X \backslash\{a, b\}} \mathscr{N}(A ; F(x)) .
$$

Следовательно,

$$
\mathbf{Z} \in \bigcup_{X \backslash\{a\}}\left(\bigcap_{x \in X \backslash\{a, b\}} \mathscr{N}(A ; F(x))\right) .
$$

В силу произвольного выбора толстой точки $C$ с непустым $\mathscr{N}(A ; C)$ и ультрафильтра $\mathbf{Z} \in \mathscr{N}(A ; C)$ включение (3.4.2) справедливо и в этой ситуации.

Теорема доказана.

В семействе всех ультрафильтров на множестве $A=F(a), a \in X$, полученная формула (3.4.1) позволяет изобразить область примыкания толстой точки $A$ к остальным толстым точкам в виде цветка «ромашки». Множество ультрафильтров

$$
S_{a}:=\bigcap_{b \in X \backslash\{a\}}\left(\bigcap_{x \in X \backslash\{a, b\}} \mathscr{N}(F(a) ; F(x))\right)=\bigcap_{x \in X \backslash\{a\}} \mathscr{N}(F(a) ; F(x))
$$

образует «сердцевину ромашки». Каждой точке $b \in X \backslash\{a\}$ соответствует «лепесток ромашки», образованный множеством ультрафильтров

$$
L_{a, b}:=\left(\bigcap_{x \in X \backslash\{a, b\}} \mathscr{N}(F(a) ; F(x))\right) \backslash \mathscr{N}(F(a) ; F(b)) .
$$

При этом область примыкания (3.4.1) есть дизъюнктное объединение

$$
\bigcup_{x \in X \backslash\{a\}} \mathscr{N}(F(a) ; F(x))=\left(\bigsqcup_{b \in X \backslash\{a\}} L_{a, b}\right) \sqcup S_{a},
$$


в котором множества могут быть пустыми.

ЗАмечание. Равенство (3.4.3) следует из того, что $X$ содержит более двух точек, поэтому $X \backslash\{a\}=\bigcup_{b \in X \backslash\{a\}}(X \backslash\{a, b\})$. Остается применить формулу для пересечений в [10, гл. $2, \S 4.2$, предложение 2].

\section{$\S$ 4. Пример}

Построим пример, демонстрирующий возможность реализации любой из возможных комбинаций в формуле (3.4.5) для области примыкания.

Пусть множество $X$ содержит не менее четырех точек. Добавив к нему «внешнюю» точку $\theta$, отметим некоторое непустое подмножество $J \subset X^{*}:=$ $X \sqcup\{\theta\}$. Обозначив через $\mathbf{N}$ множество натуральных чисел, рассмотрим прямое произведение $Y:=X \times \mathbf{N} \times J$, точки которого записываем в виде $x_{n, j}$, где $x \in X$, $n \in \mathbf{N}, j \in J$. Многозначное гиперинъективное отображение $F: X \rightarrow 2^{Y}$ задаем формулой $F(x)=\{x\} \times \mathbf{N} \times J$.

Для $n \in \mathbf{N}$ и $j \in J$ на множестве $X$ введем полуметрики

$$
\rho_{n, j}(x, y):= \begin{cases}1 / n & \text { при } j \notin\{x, y\}, x \neq y, \\ 1 & \text { при } j \in\{x, y\}, x \neq y,\end{cases}
$$

и рассмотрим на $Y$ полуметрику $\sigma$, заданную формулой

$$
\sigma\left(x_{n, j}, y_{m, i}\right):= \begin{cases}1 & \text { при }(n, j) \neq(m, i) ; x, y \in X ; x \neq y, \\ \rho_{n, j}(x, y) & \text { при } m=n, j=i \in J ; x, y \in X .\end{cases}
$$

Непосредственная (но кропотливая) проверка показывает, что $\sigma$ является птолемеевой метрикой на $Y$, при этом для любой тетрады $(a, b ; c, d)$ в $X$ выполняется оценка

$$
\alpha(F(a), F(b) ; F(c), F(d)) \geq 1 / 2 \geq(1 / 2) \alpha(a, b ; c, d)
$$

при любой птолемеевой метрике $\rho$ в $X$. Таким образом, отображение $F: X \rightarrow$ $2^{Y}$ имеет свойство $\eta$-BAD с контрольной функцией $\eta(t)=t / 2$.

Фиксируем $a \in X$ и найдем в толстой точке $F(a)$ области примыкания к ней остальных толстых точек $F(x), x \in X \backslash\{a\}$, в зависимости от задания непустого множества $J \subset X \sqcup\{\theta\}$. Все дальнейшие утверждения в этом параграфе относятся только к рассматриваемому примеру.

4.1. Утверждение. Толстая точка $F(x)$ примыкает к $F(a)$ в том и только в том случае, когда $J \backslash\{a, x\} \neq \varnothing$.

ДокАзАтЕльство. Если существует $j^{\prime} \in J \backslash\{a, x\}$, то

$$
\inf _{n, m \in \mathbf{N}}\left(\inf _{j, i \in J} \sigma\left(a_{n, j}, x_{m, i}\right)\right)=\inf _{n \in \mathbf{N}}\left(\inf _{j \in J} \rho_{n, j}(a, x)\right) \leq \inf _{n \in \mathbf{N}} \rho_{n, j^{\prime}}(a, x)=\inf _{n}(1 / n)=0
$$

и $F(x)$ примыкает к $F(a)$.

Если $J \backslash\{a, x\}=\varnothing$, то

$$
\inf _{n, m \in \mathbf{N}}\left(\inf _{j, i \in J} \sigma\left(a_{n, j}, x_{m, i}\right)\right)=\inf _{n \in \mathbf{N}}\left(\inf _{j \in J} \rho_{n, j}(a, x)\right)=\inf _{n \in \mathbf{N}}(1)=1
$$

и $F(x)$ не примыкает к $F(a)$. Утверждение доказано.

Для непустого подмножества $T \subset J \backslash\{a\}$ обозначаем через $\mathscr{N}_{T}$ множество всех ультрафильтров в $F(a)$, мажорирующих фильтр $R_{T}$ с базисом $(\{a\} \times\{n$ : $n>k\} \times T)_{k=1,2, \ldots}$. В случае бесконечного множества $J$ символом $\mathscr{N}_{\{\infty\}}$ обозначаем множество всех ультрафильтров в $F(a)$, мажорирующих фильтр $R_{\infty}$, базисом которого служит семейство множеств вида $\{a\} \times\{n: n>k\} \times(J \backslash K)$, где $k \in \mathbf{N}$ и $K \subset J-$ произвольное конечное подмножество. 
4.2. Утверждение. Если $x \in X \backslash\{a\}$ и $J \backslash\{a, x\} \neq \varnothing$, то базис фильтра $R_{\sigma}(F(a) ; F(x))$ образован множествами

$$
U_{\sigma}^{1 / k}(F(a) ; F(x))=\{a\} \times\{n: n>k\} \times(J \backslash\{a, x\})
$$

и, следовательно,

$$
\mathscr{N}(F(a) ; F(x))=\mathscr{N}_{J \backslash\{a, x\}} \cdot
$$

ДокАЗАТЕЛЬСтво. По утверждению 4.1 толстая точка $F(x)$ примыкает к $F(a)$, поэтому множества в (4.2.1) непустые. Базис фильтра $R_{\sigma}(F(a) ; F(x))$ в $F(a)$ образован множествами

$$
\begin{aligned}
& U_{\sigma}^{1 / k}(F(a) ; F(x))=\left\{a_{n, j}: \sigma\left(a_{n, j}, F(x)\right)<1 / k\right\} \\
&=\left\{a_{n, j}:(\exists(m, i)) \sigma\left(a_{n, j}, x_{m, i}\right)<1 / k\right\} \\
&=\left\{a_{n, j}: \sigma\left(a_{n, j}, x_{n, j}\right)=\rho_{n, j}(a, x)=1 / n<1 / k\right\} \\
&=\left\{a_{n, j}: n>k, j \in J \backslash\{a, x\}\right\}=\{a\} \times\{n: n>k\} \times(J \backslash\{a, x\}) .
\end{aligned}
$$

У тверждение доказано.

4.3. Утверждение. Пусть $\varnothing \neq T \subset J$ и реализуется один из следующих случаев:

(v1) $T$ - конечное множество;

(v2) $T-$ бесконечное множество, но $J \backslash T-$ конечное множество.

Тогда

$$
\mathscr{N}_{T}=\left(\bigsqcup_{t \in T} \mathscr{N}_{\{t\}}\right) \sqcup \begin{cases}\varnothing & \text { в случае }(\mathrm{v} 1), \\ \mathscr{N}_{\{\infty\}} & \text { в случае (v2), }\end{cases}
$$

и если $T$ не является одноточечным множеством, т. е. $\#(T) \geq 2$, то

$$
\left(\bigcap_{t \in T} \mathscr{N}_{T \backslash\{t\}}\right)= \begin{cases}\varnothing & \text { в случае (v1), } \\ \mathscr{N}_{\{\infty\}} & \text { в случае (v2). }\end{cases}
$$

ДокАЗАтЕльство. Элемент $\{a\} \times\{n: n>k\} \times\{z\}$ базиса фильтра $R_{\{z\}}$ не пересекается с элементами $\{a\} \times\{n: n>k\} \times\{w\}($ при $w \neq z)$ и $\{a\} \times\{n: n>$ $k\} \times(J \backslash\{z\})$ (в случае $(\mathrm{v} 2))$ базисов фильтров $R_{\{w\}}$ и $R_{\infty}$ соответственно. Поэтому не существует ультрафильтра, мажорирующего оба фильтра $R_{\{z\}}$ и $R_{\{w\}}$ или оба фильтра $R_{\{z\}}$ и $R_{\infty}$, т. е. $\mathscr{N}_{\{z\}} \cap \mathscr{N}_{\{w\}}=\varnothing$ при $z \neq w$ и $\mathscr{N}_{\{z\}} \cap \mathscr{N}_{\{\infty\}}=\varnothing$ в случае (v2). Таким образом, все множества в правой части (4.3.1) попарно не пересекаются и их объединение является дизъюнктным объединением.

Любой элемент $\{a\} \times\{n: n>k\} \times T$ базиса фильтра $R_{T}$ содержит элемент $\{a\} \times\{n: n>k\} \times\{t\}$ базиса фильтра $R_{\{t\}}$ при любом $t \in T$ и в случае (v2) (где $T=J \backslash L$ с конечным множеством $L$ ) является элементом $\{a\} \times\{n: n>$ $k\} \times(J \backslash L)$ базиса фильтра $R_{\infty}$. Это означает, что фильтр $R_{T}$ мажорируется фильтром $R_{\{t\}}$ при любом $t \in T$ и в случае (v2) мажорируется фильтром $R_{\infty}$, т. е. $\mathscr{N}_{\{t\}} \subset \mathscr{N}_{T}$ при любом $t \in T$ и в случае $(\mathrm{v} 2) \mathscr{N}_{\{\infty\}} \subset \mathscr{N}_{T}$. Это доказывает включение

$$
\mathscr{N}_{T} \supset\left(\bigsqcup_{t \in T} \mathscr{N}_{\{t\}}\right) \sqcup \begin{cases}\varnothing & \text { в случае (v1), } \\ \mathscr{N}_{\{\infty\}} & \text { в случае (v2). }\end{cases}
$$

Остается доказать обратное включение. Имеем

$$
\mathscr{N}_{T} \subset\left(\bigsqcup_{t \in T} \mathscr{N}_{\{t\}}\right) \sqcup \begin{cases}\varnothing & \text { в случае }(\mathrm{v} 1), \\ \mathscr{N}_{\{\infty\}} & \text { в случае (v2). }\end{cases}
$$


Пусть $\mathbf{Z} \in \mathscr{N}_{T}$, т. е. $\mathbf{Z}$ мажорирует фильтр $R_{T}$. Допустим, что $\mathbf{Z}$ не мажорирует ни один из фильтров $R_{\{t\}}, t \in T$. Тогда для каждого $t \in T$ найдется $k(t) \in \mathbf{N}$ такое, что элемент $\{a\} \times\{n: n>k(t)\} \times\{t\}$ базиса фильтра $R_{\{t\}}$ не входит в Z Z. По основному свойству ультрафильтров (см. [9, гл. $1, \S 6.4$, предложение 5] $F(A) \backslash(\{a\} \times\{n: n>k(t)\} \times\{t\}) \in \mathbf{Z}$. Тогда для произвольного непустого конечного $L_{0} \subset T$ и $k\left(L_{0}\right):=\max \left\{k(t): t \in L_{0}\right\}$, большее множество $F(A) \backslash\left(\{a\} \times\left\{n: n>k\left(L_{0}\right)\right\} \times\{t\}\right)$ также входит в $\mathbf{Z}$ при любом $t \in L_{0}$. Отсюда следует, что

$$
\begin{aligned}
\bigcap_{t \in L_{0}}(F(A) \backslash(\{a\} \times\{n: n> & \left.\left.\left.k\left(L_{0}\right)\right\} \times\{t\}\right)\right) \\
& =F(A) \backslash\left(\{a\} \times\left\{n: n>k\left(L_{0}\right)\right\} \times L_{0}\right) \in \mathbf{Z}
\end{aligned}
$$

при любом выборе непустого конечного множества $L_{0} \subset T$.

В случае (v1) при $L_{0}=T$ элемент $\{a\} \times\{n: n>k(T)\} \times T$ базиса фильтра $R_{T}$ не входит в $\mathbf{Z}$ и это противоречит выбору ультрафильтра $\mathbf{Z} \in \mathscr{N}_{T}$. Таким образом, в случае (v1) $\mathbf{Z}$ мажорирует один из фильтров $R_{\{t\}}, t \in T$, поэтому $\mathbf{Z} \in \bigcup_{t \in T} \mathscr{N}_{\{t\}}$, что дает требуемое включение (4.3.4).

Продолжим рассуждение для случая (v2): $T=J \backslash L$ с конечным множеством $L \subset J$. Допустим, что $\mathbf{Z}$ не мажорирует также и фильтр $R_{\infty}$. Тогда для некоторого $k_{1} \in \mathbf{N}$ и конечного множества $L_{1} \subset J$ элемент $\{a\} \times\{n: n>$ $\left.k_{1}\right\} \times\left(J \backslash L_{1}\right)$ базиса фильтра $R_{\infty}$ не входит в $\mathbf{Z}$. Пусть непустое конечное множество $L_{0} \subset T$ таково, что $\left(L_{1} \cap T\right) \subset L_{0} \subset T$ и $k^{\prime}=\max \left\{k_{1}, k\left(L_{0}\right)\right\}$. Тогда множество

$$
\begin{aligned}
\{a\} \times\left\{n: n>k^{\prime}\right\} \times\left(T \backslash L_{0}\right) & \subset\{a\} \times\left\{n: n>k_{1}\right\} \\
& \times\left(J \backslash\left(L_{1} \cap T\right)\right) \subset\{a\} \times\left\{n: n>k_{1}\right\} \times\left(J \backslash L_{1}\right)
\end{aligned}
$$

не входит в $\mathbf{Z}$. Так как в силу (4.3.5) множество

$$
\{a\} \times\left\{n: n>k^{\prime}\right\} \times L_{0} \subset\{a\} \times\left\{n: n>k\left(L_{0}\right)\right\} \times L_{0}
$$

также не входит в $\mathbf{Z}$, их объединение $\{a\} \times\left\{n: n>k^{\prime}\right\} \times T$ не входит в $\mathbf{Z}$. Это противоречит тому, что $\mathbf{Z}$ мажорирует фильтр $R_{T}$. Таким образом, в случае (v2) ультрафильтр $\mathbf{Z}$ мажорирует либо один из фильтров $R_{\{t\}}$ с $t \in T$, либо фильтр $R_{\infty}$, т. е. $\mathbf{Z} \in\left(\bigcup_{t \in T} \mathscr{N}_{\{t\}}\right) \cup \mathscr{N}_{\{\infty\}}$. Это доказывает включение (4.3.4) в случае (v2).

Из (4.3.3) и (4.3.4) получаем требуемое равенство (4.3.1).

Из (4.3.1) следует, что $\mathscr{N}_{T \backslash\{t\}}=\mathscr{N}_{T} \backslash \mathscr{N}_{\{t\}}$ при любом $t \in T$, поэтому

$$
\bigcap_{t \in T} \mathscr{N}_{T \backslash\{t\}}=\bigcap_{t \in T}\left(\mathscr{N}_{T} \backslash \mathscr{N}_{\{t\}}\right)=\mathscr{N}_{T} \backslash\left(\bigsqcup_{t \in T} \mathscr{N}_{\{t\}}\right)= \begin{cases}\varnothing & \text { в случае (v1), } \\ \mathscr{N}_{\{\infty\}} & \text { в случае (v2). }\end{cases}
$$

Утверждение доказано.

4.4. Теорема. При любом непустом множестве $J \subset X \sqcup\{\theta\}$ и любом выборе $a \in X$ область примыкания толстой точки $F(a)$ к остальным толстым точкам в $Y$ имеет вид

$$
\bigcup_{x \in X \backslash\{a\}} \mathscr{N}(F(a) ; F(x))=\left(\bigsqcup_{b \in X \backslash\{a\}} L_{a, b}\right) \sqcup S_{a},
$$


где

$$
S_{a}=\left\{\begin{array} { l l } 
{ \varnothing } & { \text { при } \theta \notin J , } \\
{ \mathscr { N } _ { \theta } } & { \text { при } \theta \in J }
\end{array} \sqcup \left\{\begin{array}{ll}
\varnothing, & \text { если } J-\text { конечное множество, } \\
\mathscr{N}_{\{\infty\}}, & \text { если } J-\text { бесконечное множество, }
\end{array}\right.\right.
$$

и для любого $b \in X \backslash\{a\}$

$$
L_{a, b}= \begin{cases}\varnothing & \text { при } b \notin J, \\ \mathscr{N}_{\{b\}} & \text { при } b \in J .\end{cases}
$$

ДокАЗАТЕЛЬСтво. Вначале рассмотрим исключительные случаи при задании конечного множества $J$.

СлучАй $J \backslash\{a\}=\varnothing$. Для любого $x \in X \backslash\{a\}$ имеем $J \backslash\{a, x\}=\varnothing$, $\mathscr{N}(F(a) ; F(x))=\varnothing$ по утверждению 4.1; область примыкания в (4.4.1) - пустое множество; $S_{a}=\varnothing=L_{a, b}$ для любого $b \in X \backslash\{a\}$.

СлучАй $J \backslash\{a\}=\left\{x_{0}\right\}, x_{0} \in X \backslash\{a\}$. В этом случае $J \backslash\left\{a, x_{0}\right\}=\varnothing$ и $\mathscr{N}\left(F(a) ; F\left(x_{0}\right)\right)=\varnothing$ (утверждение 4.1$) ; \mathscr{N}(F(a) ; F(x))=\mathscr{N}_{\left\{x_{0}\right\}}$ при $x \in X \backslash$ $\left\{a, x_{0}\right\}$ (утверждение 4.2); левая часть в (4.4.1) есть $\mathscr{N}_{\left\{x_{0}\right\}} ; S_{a}=\varnothing ; L_{a, x_{0}}=\mathscr{N}_{\left\{x_{0}\right\}}$ и $L_{a, b}=\varnothing$ при $b \in X \backslash\left\{a, x_{0}\right\}$.

СлучАй $J \backslash\{a\}=\{\theta\}$. Для любого $x \in X \backslash\{a\}$ имеем: $J \backslash\{a, x\}=\{\theta\}$ и $\mathscr{N}(F(a) ; F(x))=\mathscr{N}_{\{\theta\}}$ (утверждение 4.2); область примыкания в (4.4.1) есть $\mathscr{N}_{\{\theta\}} ; S_{a}=\mathscr{N}_{\{\theta\}} ; L_{a, b}=\mathscr{N}_{\{\theta\}} \backslash \mathscr{N}_{\{\theta\}}=\varnothing$ при любом $b \in X \backslash\{a\}$.

Так как во всех этих случаях формулы (4.4.1)-(4.4.3) истинны, в дальнейшем доказательстве считаем, что $J \backslash\{a\}$ содержит более одной точки.

При вычислении $S_{a}$ по формуле (3.4.3) заметим, что $\theta \notin X$ и потому

$$
S_{a}=\bigcap_{x \in X \backslash\{a\}} \mathscr{N}(F(a) ; F(x))=\bigcap_{x \in X \backslash\{a, \theta\}} \mathscr{N}(F(a) ; F(x)) .
$$

Используя формулы (4.2.1) и (4.3.1), получаем равенство

$$
\begin{aligned}
& \bigcap_{x \in J \backslash\{a, \theta\}} \mathscr{N}(F(a) ; F(x))=\bigcap_{x \in J \backslash\{a, \theta\}} \mathscr{N}_{J \backslash\{a, x\}} \\
& =\bigcap_{x \in J \backslash\{a, \theta\}}\left(\mathscr{N}_{J \backslash\{a\}} \backslash \mathscr{N}_{\{x\}}\right)=\mathscr{N}_{J \backslash\{a\}} \backslash\left(\bigsqcup_{x \in J \backslash\{a, \theta\}} \mathscr{N}_{\{x\}}\right) \\
& =\left[\left(\bigsqcup_{x \in J \backslash\{a, \theta\}} \mathscr{N}_{\{x\}}\right) \sqcup\left\{\begin{array}{ll}
\varnothing & \text { при } \theta \notin J, \\
\mathscr{N}_{\{\theta\}} & \text { при } \theta \in J
\end{array}\right\} \sqcup\left\{\begin{array}{ll}
\varnothing & \text { при } \# J<\infty, \\
\mathscr{N}_{\{\infty\}} & \text { при } \# J=\infty
\end{array}\right\}\right] \\
& \backslash\left(\bigsqcup_{x \in J \backslash\{a, \theta\}} \mathscr{N}_{\{x\}}\right)=\left\{\begin{array}{ll}
\varnothing & \text { при } \theta \notin J, \\
\mathscr{N}_{\{\theta\}} & \text { при } \theta \in J
\end{array}\right\} \sqcup\left\{\begin{array}{ll}
\varnothing & \text { при } \# J<\infty, \\
\mathscr{N}_{\{\infty\}} & \text { при } \# J=\infty
\end{array}\right\} .
\end{aligned}
$$

Для любой точки $x \in P:=(X \backslash\{a, \theta\}) \backslash(J \backslash\{a, \theta\})$ (если $P \neq \varnothing)$ имеем равенство $\mathscr{N}(F(a) ; F(x))=\mathscr{N}_{J \backslash\{a, x\}}=\mathscr{N}_{J \backslash\{a\}}$. Поэтому при $P \neq \varnothing$ в силу (4.4.4) выполняется включение

$$
\bigcap_{x \in P} \mathscr{N}(F(a) ; F(x))=\mathscr{N}_{J \backslash\{a\}} \supset \bigcap_{x \in J \backslash\{a, \theta\}} \mathscr{N}(F(a) ; F(x)) .
$$

Значит, в любом из случаев $P=\varnothing$ и $P \neq \varnothing$ получаем равенство

$$
S_{a}=\bigcap_{x \in J \backslash\{a, \theta\}} \mathscr{N}(F(a) ; F(x))
$$


и тогда формула (4.4.4) дает требуемое равенство (4.4.2).

Если $b \notin J$, то $J \backslash\{a, b\}=J \backslash\{a\} \neq \varnothing ; \mathscr{N}(F(a) ; F(b))=\mathscr{N}_{J \backslash\{a, b\}}=\mathscr{N}_{J \backslash\{a\}}$ (см. (4.2.1)); $\mathscr{N}(F(a) ; F(x))=\mathscr{N}_{J \backslash\{a, x\}} \subset \mathscr{N}_{J \backslash\{a\}}$ (см. (4.3.1)) для любого $x \in X \backslash\{a, b\} \neq \varnothing$. Поэтому

$$
\begin{gathered}
\bigcap_{x \in X \backslash\{a, b\}} \mathscr{N}(F(a) ; F(x)) \subset \mathscr{N}(F(a) ; F(b)), \\
L_{a, b}=\bigcap_{x \in X \backslash\{a, b\}} \mathscr{N}(F(a) ; F(x)) \backslash \mathscr{N}(F(a) ; F(b))=\varnothing,
\end{gathered}
$$

т. е. в этом случае (4.4.3) выполняется.

Пусть $b \in J$. Так как $\#(J \backslash\{a\})>1$, то $J \backslash\{a, b\} \neq \varnothing$ и

$$
\mathscr{N}(F(a) ; F(b))=\mathscr{N}_{J \backslash\{a, b\}}=\mathscr{N}_{J \backslash\{a\}} \backslash \mathscr{N}_{\{b\}} .
$$

Если $J \backslash\{a, b, \theta\}=\varnothing$, то $J \subset\{a, b, \theta\}$ и $x \notin J$ при любом $x \in X \backslash\{a, b\}$. В этом случае формула (4.4.3) верна, ибо

$$
\bigcap_{x \in X \backslash\{a, b\}} \mathscr{N}(F(a) ; F(x))=\bigcap_{x \in X \backslash\{a, b\}} \mathscr{N}_{J \backslash\{a, x\}}=\mathscr{N}_{J \backslash\{a\}}
$$

и в силу (4.4.5) $L_{a, b}=\mathscr{N}_{J \backslash\{a\}} \backslash\left(\mathscr{N}_{J \backslash\{a\}} \backslash \mathscr{N}_{\{b\}}\right)=\mathscr{N}_{\{b\}}$.

Наконец, пусть $J \backslash\{a, b, \theta\} \neq \varnothing$ и $c \in J \backslash\{a, b, \theta\}$. Для любого $x \in P:=$ $(X \backslash\{a, b\}) \backslash(J \backslash\{a, b, \theta\})$ (если $P \neq \varnothing)$ имеем включение $\mathscr{N}(F(a) ; F(x))=$ $\mathscr{N}_{J \backslash\{a, x\}}=\mathscr{N}_{J \backslash\{a\}} \supset \mathscr{N}_{J \backslash\{a, c\}}$. Поэтому в любом из случаев $P=\varnothing$ или $P \neq \varnothing$ справедливо равенство

$$
\bigcap_{x \in X \backslash\{a, b\}} \mathscr{N}(F(a) ; F(x))=\bigcap_{x \in J \backslash\{a, b, \theta\}} \mathscr{N}(F(a) ; F(x))=\bigcap_{x \in J \backslash\{a, b, \theta\}} \mathscr{N}_{J \backslash\{a, x\}} .
$$

Для любых множеств $A, B, C$ справедливо равенство $(A \backslash B) \backslash(A \backslash C)=(A \cap C) \backslash B$. Поэтому

$$
\begin{gathered}
L_{a, b}=\left(\bigcap_{x \in J \backslash\{a, b, \theta\}}\left(\mathscr{N}_{J \backslash\{a\}} \backslash \mathscr{N}_{\{x\}}\right)\right) \backslash\left(\mathscr{N}_{J \backslash\{a\}} \backslash \mathscr{N}_{\{b\}}\right) \\
=\left(\mathscr{N}_{J \backslash\{a\}} \backslash\left(\bigsqcup_{x \in J \backslash\{a, b, \theta\}} \mathscr{N}_{\{x\}}\right)\right) \backslash\left(\mathscr{N}_{J \backslash\{a\}} \backslash \mathscr{N}_{\{b\}}\right) \\
=\left(\mathscr{N}_{J \backslash\{a\}} \cap \mathscr{N}_{\{b\}}\right) \backslash\left(\bigsqcup_{x \in J \backslash\{a, b, \theta\}} \mathscr{N}_{\{x\}}\right)=\mathscr{N}_{\{b\}} \backslash\left(\bigsqcup_{x \in J \backslash\{a, b, \theta\}} \mathscr{N}_{\{x\}}\right)=\mathscr{N}_{\{b\}},
\end{gathered}
$$

ибо $\left(\bigcup_{x \in J \backslash\{a, b, \theta\}} \mathscr{N}_{\{x\}}\right) \cap \mathscr{N}_{\{b\}}=\varnothing$. Итак, формула (4.4.3) верна и в этом случае. Теорема доказана.

ЗАмечаниЕ. В [4] в формуле (0.3) замечена опечатка: вместо $\alpha(F(\Psi)) \geq$ $\alpha(\Psi)$ следует читать $\alpha(F(\Psi)) \geq \omega(\alpha(\Psi))$.

Благодарность. Автор чрезвычайно благодарен рецензенту, отметившему ряд неточностей и опечаток. 


\section{ЛИТЕРАТУРА}

1. Асеев В. В. Многозначные отображения со свойством квазимёбиусовости // Сиб. мат. журн. 2019. Т. 60, № 5. С. 953-972.

2. Асеев В. В., Сычёв А. В., Тетенов А. В. Мёбиусово-инвариантные метрики и обобщенные углы в птолемеевых пространствах // Сиб. мат. журн. 2005. Т. 46, № 2. С. 243-263.

3. Асеев В. В. Обобщенные углы в птолемеевых мёбиусовых структурах // Сиб. мат. журн. 2018. T. 59, № 2. C. 241-256.

4. Асеев В. В. Обобщенные углы в птолемеевых мёбиусовых структурах. II // Сиб. мат. журн. 2018. Т. 59, № 5. С. 976-987.

5. Aseev V. V. On coordinate vector-functions of quasiregular mappings // Sib. Electron. Math. Rep. 2018. V. 15. P. 768-772.

6. Väisälä J. Quasimöbius maps // J. Anal. Math. 1984/85. V. 44. P. 218-234.

7. Решетняк Ю. Г. Пространственные отображения с ограниченным искажением. Новосибирск: Наука, 1982.

8. Буяло С. В. Мёбиусовы и субмёбиусовы структуры // Алгебра и анализ. 2016. Т. 28, № 5. C. $1-20$.

9. Бурбаки Н. Общая топология. Основные структуры. М.: Наука, 1968.

10. Бурбаки Н. Теория множеств. М.: Мир, 1965.

Поступила в редакиию 8 октября 2019 г.

После доработки 4 февраля 2020 г.

Принята к публикачии 19 февраля 2020 г.

Асеев Владислав Васильевич

Институт математики им. С. Л. Соболева СО РАН, пр. Академика Коптюга, 4, Новосибирск 630090

btp@math.nsc.ru 